\title{
HOMOLOGICAL DIMENSION AND THE CONTINUUM HYPOTHESIS
}

\author{
B. L. OSOFSKY ${ }^{(1)}$
}

1. Introduction. In [9], Kaplansky proves:

Let $R$ be a commutative local domain with quotient field $Q$. Then the following are equivalent:

(i) The homological dimension of $Q_{R}, \mathrm{hd}_{R}(Q),=1$.

(ii) $Q$ is countably generated.

In [14], Small shows that the hypothesis " $R$ local" may be replaced either by " $R$ is domain such that the Jacobson radical $J \neq 0$ and $R / J$ is Noetherian" or by " $R$ is a regular domain which is not Dedekind, such that $R$ contains an uncountable field," and (i) will still be equivalent to (ii). Small also shows that, if $Q$ is generated by $\aleph_{n}$ elements, $n$ an integer, then $h_{R}(Q) \leqq n+1$. This implies that, for $Q_{R}$ countably generated, $\mathrm{hd}_{R}(Q)=1$. This special case also appears in Matlis [10].

The question naturally arises if, for all commutative domains $R, \operatorname{hd}_{R}(Q)=n+1$ if any generating set for $Q_{R}$ has at least $\boldsymbol{\aleph}_{n}$ elements and the global dimension of $R>n$. By Matlis [10], the answer is no-a Noetherian domain of Krull dimension 1 always has $\operatorname{hd}_{R}(Q)=1$. However, for regular domains, a situation of particular interest to Small in [14], we obtain some information. By means of the techniques of Osofsky [13] and Kaplansky [9], we show:

Let $R$ be a regular local ring of dimension $m$ such that cardinality $R=$ cardinality $R / J$ or $R$ is complete. Let $Q$ be generated by a set of $\boldsymbol{\aleph}_{k}$ but no fewer elements. Then $\operatorname{hd}_{R}(Q)=\min \{k+1, m\}$. This is then generalized to regular domains finitely generated over some field. As indicated in Small [14], this gives a new statement equivalent to the continuum hypothesis. Indeed, for $n \in \omega$, let $F_{m}$ be the ring of polynomials in $m \geqq n+3$ variables over a field $F$ with cardinality $2^{\aleph_{n}}$. Let $Q_{m}$ be its quotient field. Then $\mathrm{hd}_{F_{m}}\left(Q_{m}\right)=n+2 \Leftrightarrow 2 \aleph_{n}=\boldsymbol{\aleph}_{n+1}$. In particular, if $\boldsymbol{R}$ is the field of real numbers, $\operatorname{hd}_{R_{3}}\left(Q_{3}\right)=2 \Leftrightarrow$ the continuum hypothesis holds. One can actually write down a module over $\boldsymbol{R}_{3}$ which is free $\Leftrightarrow 2 \boldsymbol{\aleph}_{0}=\boldsymbol{\aleph}_{1}$. Let $F$ be the free $\boldsymbol{R}_{3}$-module generated by $\boldsymbol{R}_{3} \times \boldsymbol{R}_{3}$, i.e. $F=\sum(x, y) \in \boldsymbol{R}_{3} \times \boldsymbol{R}_{3} \oplus(x, y) \boldsymbol{R}_{3}$. Let $M$ be the submodule of $F$ generated by $\{(y, z)-(x, z)+(x, y) y / z \mid y=z a, x=y b$, where $a$ and $b$ are nonconstant polynomials $\}$. Then $M$ is projective $\Leftrightarrow$ the continuum hypothesis holds. Since $M$ is infinitely generated, by a result of Bass [4], $M$ is free $\Leftrightarrow M$ is projective. In the appendix, we show how to construct a free basis for $M$ if the continuum hypothesis

Received by the editors March 2, 1967 and, in revised form, August 10, 1967.

(1) The author gratefully acknowledges financial support from the National Science Foundation under grant GP-4226. 
holds. If one changes $\boldsymbol{R}_{3}$ to $\boldsymbol{R}_{2}$, the corresponding module always has a free basis, so we see no way to show directly from the module $M$ that if $2 \mathrm{~N}_{0} \neq \mathrm{\aleph}_{1}, M$ cannot have a free basis.

Most of the results in Small [14] and Osofsky [13, §2] are special cases of generalizations proved here. One might hope that the techniques employed could be combined with appropriate induction hypotheses to yield dimensions of other modules over other rings.

2. Definitions and notation. Throughout this paper, $R$ will denote a ring with 1 . All modules will be unital right $R$-modules.

$|A|$ will denote the cardinality of the set $A$.

Let $A, B$, and $C$ be $R$-modules, $\lambda: A \rightarrow C, \mu: B \rightarrow C, \nu: A \rightarrow B R$-homomorphisms. $(\lambda, v): A \rightarrow C \oplus B$ will denote the homomorphism defined by $(\lambda, \nu) a=\lambda a+\nu a$ for all $a \in A . \lambda \oplus \mu: A \oplus B \rightarrow C$ is defined by $(\lambda \oplus \mu)(a+b)$ $=\lambda a+\mu b$ for all $a \in A, b \in B$.

A right $R$-module $M$ will be called directed if :

(i) $M$ is generated by a set of elements $M^{\prime}$ such that $x r=0 \Leftrightarrow r=0$ for all $x \in M^{\prime}$.

(ii) For all $x, y \in M^{\prime}$, there exists a $z \in M^{\prime}$ such that $z R \supseteq x R+y R$.

$M^{\prime}$ will be called a set of free generators for $M$.

If $M$ is a directed module with free generators $M^{\prime}, u: M^{\prime} \times M^{\prime} \rightarrow M^{\prime}$ is called an upper bound function if $u(x, y) R \supseteq x R+y R$ for all $x, y \in M^{\prime}$. We extend $u$ to a function from $\bigcup_{n=2}^{\infty}\left(M^{\prime}\right)^{n}$ to $M^{\prime}$ inductively by

$$
u\left(m_{1}, \ldots, m_{n}\right)=u\left(m_{1}, u\left(m_{2}, \ldots, m_{n}\right)\right)
$$

Then $u\left(m_{1}, \ldots, m_{n}\right) R \supseteq \sum_{i=1}^{n} m_{i} R$. If $X \subseteq M^{\prime}$ and $u(X \times X) \subseteq X, X$ will be called $u$-closed. For all $Y \subseteq M^{\prime}$, define the $u$-closure of $Y, \mathrm{cl}(Y)$, by

$$
\operatorname{cl}(Y)=\underset{Y \subseteq X \subseteq M^{\prime} ; X u \text {-closed }}{ } X .
$$

If we set $Y_{0}=Y, Y_{n+1}=Y_{n} \cup u\left(Y_{n} \times Y_{n}\right)$, then $\operatorname{cl}(Y)=\bigcup_{n=0}^{\infty} Y_{n}$.

We observe:

2.1 If $|Y| \geqq \aleph_{0}$, then $|\mathrm{cl}(Y)|=|Y|$ since $\left|Y_{n+1}\right|=\left|Y_{n}\right|$ for all $n \geqq 0$.

2.2 If $X$ is $u$-closed, the submodule of $M$ generated by $X$ is directed.

2.3 If $M$ is directed, then $M$ is finitely generated $\Leftrightarrow M$ is cyclic.

2.4 If $M$ is directed and $X$ is a set of free generators for $M$, then $X$ is directed by inclusion of cyclic submodules.

2.5 If $M$ is directed and countably generated, then $M=\bigcup_{i=0}^{\infty} x_{i} R$ where $x_{i} R$ is free and $x_{i} R \subseteq x_{j} R$ for all $i \leqq j$.

$\mathrm{hd}_{R}(M)$ will denote the homological dimension of $M_{R}$. If $x, y \in M, x \leqq y$ will mean $x R \subseteq y R$. If $M$ is directed, $x \in M^{\prime}, x^{-1}$ will denote the $R$-isomorphism: 
$x R \rightarrow R$ given by $x^{-1}(x r)=r . x^{-1}$ exists since $x R$ is free with basis $x$. Note that $x \cdot x^{-1}(x r)=x r$.

Let $X \subseteq M_{R}, n \geqq 0 . P_{n}(X)$ will denote the free $R$-module

$$
P_{n}(X)=\sum_{\left\{x_{i} \mid 0 \leqq i \leqq n\right\} \subseteq X ; x_{0}>x_{1}>\ldots>x_{n}} \oplus\left\langle x_{0}, \ldots, x_{n}\right\rangle R
$$

where, for all $r \in R,\left\langle x_{0}, \ldots, x_{n}\right\rangle r=0 \Leftrightarrow r=0$. Set $P_{-1}(X)=$ the submodule of $M$ generated by $X$.

Let $x \in M^{\prime}$. Set $s(x)=\left\{y \in M^{\prime} \mid y<x\right\}, \bar{s}(x)=\left\{y \in M^{\prime} \mid y \leqq x\right\}$. We define a map $x^{*}: P_{n}(s(x)) \rightarrow P_{n+1}(\bar{s}(x))$ for $n \geqq 0$ by

$$
x^{*}\left\langle x_{0}, \ldots, x_{n}\right\rangle=\left\langle x, x_{0}, \ldots, x_{n}\right\rangle .
$$

If $n=-1, x^{*}: P_{-1}(x) \rightarrow P_{0}(\bar{s}(x))$ is defined by

$$
x^{*}(x r)=\langle x\rangle r=\langle x\rangle x^{-1}(x r) .
$$

For $n \geqq 0$, define a function $d_{n}: P_{n}(X) \rightarrow P_{n-1}(X)$ by

$$
\begin{aligned}
d_{0}\langle x\rangle & =x, \\
d_{n}\left\langle x_{0}, \ldots, x_{n}\right\rangle & =\sum_{i=1}^{n-1}\left\langle x_{0}, \ldots, \hat{x}_{i}, \ldots, x_{n}\right\rangle(-1)^{i}+\left\langle x_{0}, \ldots, x_{n-1}\right\rangle(-1)^{n} x_{n-1}^{-1}\left(x_{n}\right)
\end{aligned}
$$

where $\hat{x}_{i}$ means delete $x_{i}$.

$x^{*}$ and $d_{i}$ are analogous to the "adjoin a vertex" and boundary operators of combinatorial topology, and are precisely the functions defined in [13]. They are connected by a basic relation:

$$
d_{n+1}\left(x^{*} p\right)=p-x^{*} d_{n} p \text { for all } n \geqq 0, p \in P_{n}(s(x)) .
$$

This relation will often be used without explicit reference to it. It is verified by direct computation.

3. The projective resolution of a directed $R$-module. We apply the argument in [13] to get a projective resolution of a directed $R$-module which we will use to calculate its dimension in special cases.

3.1 Proposition. Let $M$ be a directed R-module with set of free generators $M^{\prime}$ and upper bound function $u$. Let $X$ be a u-closed subset of $M^{\prime}$. Then

$$
\left(\mathscr{P}_{X}\right) \cdots \stackrel{d_{n+1}}{\longrightarrow} P_{n}(X) \stackrel{d_{n}}{\longrightarrow} P_{n-1}(X) \longrightarrow \cdots \stackrel{d_{1}}{\longrightarrow} P_{0}(X) \stackrel{d_{0}}{\longrightarrow} P_{-1}(X) \longrightarrow 0
$$

is a projective resolution of $P_{-1}(X)=$ the submodule generated by $X$.

Proof. (i) $\mathscr{P}_{X}$ is a complex. This is a straightforward computation, written out in [13].

(ii) $\mathscr{P}_{X}$ is exact. $\mathscr{P}_{X}$ is exact at $P_{-1}(X)$ since $X$ generates $P_{-1}(X)$. 
Let $p=\sum_{i=1}^{k}\left\langle x_{0}^{i}, \ldots, x_{n}^{i}\right\rangle r_{i} \in P_{n}(X), d_{n} p=0$. Let $x=u\left(x_{0}^{1}, \ldots, x_{0}^{k}\right)$. Assume $x_{0}^{1}, \ldots, x_{0}^{l}<x=x_{0}^{l+1}=\cdots=x_{0}^{k}$, and set $p^{\prime}=\sum_{i=1}^{l}\left\langle x_{0}^{i}, \ldots, x_{n}^{i}\right\rangle r_{i}, p^{\prime \prime}=p-p^{\prime}$. By definition, $p^{\prime \prime}=x^{*} q$ for some $q \in P_{n-(s(x))}$ or $x R$. By 2.6,

$$
p-d_{n+1}\left(x^{*} p^{\prime}\right)=x^{*} q+x^{*} d_{n} p^{\prime}
$$

Since $\mathscr{P}_{X}$ is a complex

$$
\begin{aligned}
0 & =d_{n}\left[x^{*}\left(q+d_{n} p^{\prime}\right)\right]=q+d_{n} p^{\prime}+x^{*}(d q) & & \text { if } n>0, \\
& =x x^{-1}\left(q+d_{n} p^{\prime}\right)=q+d_{n} p^{\prime} & & \text { if } n=0 .
\end{aligned}
$$

Since for $n>0$, no term of $q+d_{n} p^{\prime}$ involves the symbol $x$, and every term of $x^{*} d q$ does, $q+d_{n} p^{\prime}=0$. Hence $p=d_{n+1}\left(x^{*} p^{\prime}\right)$.

We are also interested in a projective resolution of a quotient of two directed modules.

3.2 Proposition. Let $M$ be a directed R-module, $X$ and $Y$ u-closed subsets of $M^{\prime}, X \subseteq Y$. Let $\nu$ be the natural map from $P_{-1}(Y) \rightarrow P_{-1}(Y) / P_{-1}(X), I$ the identity on $P_{n}(X)$. Then

$$
\begin{aligned}
\left(\mathscr{P}_{X, Y}\right) \cdots & \longrightarrow P_{n}(X) \oplus P_{n+1}(Y) \stackrel{\left(-d_{n}, I\right) \oplus d_{n+1}}{\longrightarrow} P_{n-1}(X) \oplus P_{n}(Y) \\
& \longrightarrow \cdots \stackrel{\left(-d_{1}, I\right) \oplus d_{2}}{\longrightarrow} P_{0}(X) \oplus P_{1}(Y) \stackrel{I \oplus d_{1}}{\longrightarrow} P_{0}(Y) \\
& \stackrel{v d_{0}}{\longrightarrow} P_{-1}(Y) / P_{-1}(X) \longrightarrow 0
\end{aligned}
$$

is a projective resolution of $P_{-1}(Y) / P_{-1}(X)$.

Proof. Clearly $\mathscr{P}_{X, Y}$ is exact at $P_{-1}(Y) / P_{-1}(X)$ since $d_{0}$ is onto $P_{-1}(Y)$. Also, $v d_{0}\left(I \oplus d_{1}\right)=0$ since $d_{0} P_{0}(X) \subseteq P_{-1}(X)$ and $d_{0} d_{1}=0$. Let $z \in$ kernel $\nu d_{0}$. Then $d_{0}(z) \in P_{-1}(X)$. Since $d_{0}: P_{0}(X) \rightarrow P_{-1}(X)$ is onto, there is an $x \in P_{0}(X)$ such that $d_{0}(x-z)=0$. Since $\mathscr{P}_{Y}$ is exact, $z \in P_{0}(X)+d_{1}\left(P_{1}(Y)\right)$ and $\mathscr{P}_{X, Y}$ is exact at $P_{0}(Y)$.

Moreover,

$$
\left[I \oplus d_{1}\right]\left[\left(-d_{1}, I\right) \oplus d_{2}\right]=\left(-d_{1}+d_{1}, d_{1} d_{2}\right)=0 .
$$

If $\left(I \oplus d_{1}\right)(a, b)=0, a \in P_{0}(X), b \in P_{1}(Y)$, then $a+d_{1} b=0$, and by the exactness of $\mathscr{P}_{X}$ and $\mathscr{P}_{Y}$, there is a $z \in P_{1}(X)$ and $w \in P_{2}(Y)$ such that $d_{1} z=d_{1} b=-a$ and $z=$ $b+d_{2} w$. Then $(a, b)=\left[\left(-d_{1}, I\right) \oplus d_{2}\right](z,-w)$ so $\mathscr{P}_{X, Y}$ is exact at $P_{0}(X) \oplus P_{1}(Y)$.

For $n>1$,

$$
\left[\left(-d_{n-1}, I\right) \oplus d_{n}\right]\left[\left(-d_{n}, I\right) \oplus d_{n+1}\right]=\left(d_{n-1} d_{n},-d_{n}+d_{n}\right) \oplus d_{n} d_{n+1}=0 .
$$

Hence $\mathscr{P}_{X, Y}$ is a complex. Let

$$
\left[\left(-d_{n}, I\right) \oplus d_{n+1}\right](a, b)=0 .
$$

Then

$$
0=-d_{n} a=a+d_{n+1} b
$$


By the exactness of $\mathscr{P}_{X}, d_{n+1}(b)=-a=d_{n+1}(z)$ for some $z \in P_{n+1}(X)$, and $b-z$ $=d_{n+2}(w)$ for some $w \in P_{n+2}(Y)$. Then $\left[\left(-d_{n+1}, I\right) \oplus d_{n+2}\right](z, w)=(a, b)$. Hence $\mathscr{P}_{X, Y}$ is exact.

Clearly every module in $\mathscr{P}_{X, Y}$ is projective (indeed free).

4. The Small inequality. We apply an argument in Small [14] to get one inequality on the dimension of a directed $R$-module.

4.1 LemMA (AUSLANDER). Let $M$ be a right $R$-module, $\mathscr{I}$ a nonempty well-ordered set, and $\left\{N_{i} \mid i \in \mathscr{I}\right\}$ a family of submodules of $M$ such that if $i, j \in \mathscr{I}$ and $i \leqq j$, then $N_{i} \subseteq N_{j}$. If $M=\bigcup_{i \in \mathscr{I}} N_{i}$ and $\mathrm{hd}_{R}\left(N_{i} / \bigcup_{j<i} N_{j}\right) \leqq n$ for all $i \in \mathscr{I}$, then $\operatorname{hd}_{R}(M) \leqq n$.

This is Proposition 3 of Auslander [1].

4.2 Proposition. Let $M$ be a directed $R$-module possessing a free generating set $X$ of $\aleph_{n}$ elements for some $n \in \omega$. Then $\operatorname{hd}_{R}(M) \leqq n+1$.

Proof. If $n=0$, by 2.5, $M=\bigcup_{i=0}^{\infty} x_{i} R$. Since $x_{i} R$ has dimension 0 for each $i$ and $0 \rightarrow x_{i} R \rightarrow x_{i+1} R \rightarrow x_{i+1} R / x_{i} R \rightarrow 0$ is exact, hd $\left(x_{i+1} R / x_{i} R\right) \leqq 1$ for each $i$. By 4.1, $\operatorname{hd}_{R}(M) \leqq 1$.

Now assume $n>0$ and the proposition holds for $n-1$. Index $X$ by $\aleph_{n}$, i.e. $X=\left\{x_{\alpha} \mid \alpha<\boldsymbol{W}_{n}\right\}$. By transfinite induction we define a set of $u$-closed subsets of $X$, $\left\{X_{\alpha} \mid \alpha<\boldsymbol{\aleph}_{n}\right\}$ such that $X_{0} \supseteq\left\{x_{\alpha} \mid \alpha \in \omega\right\}, X_{\alpha}=\bigcup_{\beta<\alpha} X_{\beta}$ for $\alpha$ a limit ordinal, and $X_{\beta+1}=\operatorname{cl}\left(X_{\beta} \cup\left\{x_{\beta}\right\}\right)$. We note $\left|X_{0}\right|<\boldsymbol{\aleph}_{n}$. Assume $\left|X_{\beta}\right|<\boldsymbol{\aleph}_{n}$ for all $\beta<\alpha$. If $\alpha$ is a limit ordinal, $X_{\alpha}$ is a union of $|\alpha|<\boldsymbol{\aleph}_{n}$ sets of cardinality $<\boldsymbol{\aleph}_{n}$, so $\left|X_{\alpha}\right|<\boldsymbol{\aleph}_{n}$. If $\alpha=\beta+1$, since $X_{\beta} \supseteq X_{0}$ which is infinite, $\left|X_{\beta} \cup\left\{x_{\beta}\right\}\right|=\left|X_{\beta}\right|<\mathcal{K}_{n}$, so by $2.1,\left|X_{\beta+1}\right|$ $=\left|X_{\beta}\right|<\boldsymbol{\aleph}_{n}$. Hence each $X_{\alpha}$ has $\left|X_{\alpha}\right|<\boldsymbol{\aleph}_{n}$ by induction. Then $M=\bigcup_{\alpha<\boldsymbol{K}_{n}} P_{-1}\left(X_{\alpha}\right)$, and by the induction hypothesis, hd $\left(P_{-1}\left(X_{\alpha}\right)\right) \leqq n$. Since $\bigcup_{\beta<\alpha} P_{-1}\left(X_{\beta}\right)=P_{-1}\left(X_{\alpha}\right)$ if $\alpha$ is a limit ordinal or $P_{-1}\left(X_{\alpha-1}\right)$ if $\alpha$ is a successor ordinal, in the exact sequence

$$
0 \rightarrow \bigcup_{\beta<\alpha} P_{-1}\left(X_{\beta}\right) \rightarrow P_{-1}\left(X_{\alpha}\right) \rightarrow P_{-1}\left(X_{\alpha}\right) / \bigcup_{\beta<\alpha} P_{-1}\left(X_{\beta}\right) \rightarrow 0
$$

two of the three terms have dimension $\leqq n$. Hence hd $\left(P_{-1}\left(X_{\alpha}\right) / \bigcup_{\beta<\alpha} P_{-1}\left(X_{\beta}\right)\right)$ $\leqq n+1$. Then $\mathrm{hd}_{R}(M) \leqq n+1$ by 4.1 .

5. Direct summands of a projective $d_{n} P_{n}$.

5.1 Lemma (KAPLANSKY). A projective module over any ring $R$ is a direct sum of countably generated submodules.

For a proof see Kaplansky [7].

Let $p \in P_{n}(X), p=\sum_{i=1}^{m}\left\langle x_{0}^{i}, \ldots, x_{n}^{i}\right\rangle r_{1}$. We say $x \in X$ appears in $p$ if $x=x_{j}^{i}$ for some $i$ and $j, 0 \leqq j \leqq n, r_{i} \neq 0$. For each $p \in P_{n}(X),\{x \in X \mid x$ appears in $p\}$ is finite. For $Y \subseteq P_{n}(X)$, set

$$
a(Y)=\{x \in X \mid x \text { appears in } p \text { for some } p \in Y\} .
$$

5.2 Proposition. Let $M$ be a directed $R$-module with free generators $M^{\prime}$, upper bound function $u$ and projective dimension $\leqq k$ such that no set of cardinality $\leqq \aleph_{n}$ 
generates $M$ for some $n \in \omega$. Let $Z \subseteq M^{\prime}$ have $|Z| \leqq \aleph_{n}$. Then there exists a u-closed set $Y \subseteq M^{\prime}$ such that $Z \subseteq Y$ and

(a) $|Y|=\boldsymbol{\aleph}_{n}$.

(b) No set of cardinality $<\aleph_{n}$ generates $P_{-1}(Y)$.

(c) $d_{k} P_{k}(Y)$ is a direct summand of $d_{k} P_{k}\left(M^{\prime}\right)$.

Proof. Since $\mathrm{hd}_{R}(M) \leqq k, d_{k} P_{k}\left(M^{\prime}\right)$ is projective. By 5.1, $d_{k} P_{k}\left(M^{\prime}\right)=\sum_{i \in \mathcal{I}} \oplus Q_{i}$ where $Q_{i}$ is countably generated. For $X \subseteq d_{k} P_{k}\left(M^{\prime}\right)$, set

$$
X^{\dagger}=\bigcap\left\{\sum_{i \in \mathscr{J}} Q_{i} \mid \mathscr{J} \subseteq \mathscr{I}, X \subseteq \sum_{i \in \mathscr{J}} Q_{i}\right\} .
$$

Let $X$ be infinite. Since each element in $X$ is in a finite sum of $Q_{i}$ 's, if $X^{\dagger}=\sum_{i \in \mathscr{X}} Q_{i}$, $|\mathscr{K}| \leqq|X|$. Since each $Q_{i}$ is countably generated, $\left|a\left(Q_{i}\right)\right| \leqq \aleph_{0}$. Then

$$
\left|a\left(X^{\dagger}\right)\right|=\left|a\left(\sum_{i \in \mathscr{X}} Q_{i}\right)\right| \leqq|\mathscr{K}| \cdot \boldsymbol{\aleph}_{0} \leqq|X| .
$$

Now let $Y_{0}$ be the $u$-closure of $Z$ (hence $\left|Y_{0}\right| \leqq \aleph_{n}$ ). We inductively define $Y_{\alpha}$ for $\alpha<\aleph_{n}$ with the following properties:

(i) $\left|Y_{\alpha}\right| \leqq \aleph_{n}$,

(ii) $Y_{\alpha}=\bigcup_{\beta<\alpha} Y_{\beta}$ for $\alpha$ a limit ordinal,

(iii) $Y_{\alpha+1}=\operatorname{cl}\left(a\left\{\left[d_{k} P_{k}\left(\mathrm{cl}\left(Y_{\alpha} \cup\{x\}\right)\right)\right]^{\dagger}\right\}\right)$ where $x \notin P_{-1}\left(Y_{\alpha}\right)$. (I.e., add an extra element to $Y_{\alpha}$, close it to get a directed set, apply $d_{k}$ to the corresponding $(k+1)$ tuples, take all elements appearing in a minimal set of $Q$ 's containing this image, close again.)

Since $\left|Y_{\alpha}\right| \leqq \aleph_{n}, P_{-1}\left(Y_{\alpha}\right) \neq M$. Hence (iii) is always possible. Since a union of $<\boldsymbol{\aleph}_{n}$ sets of cardinality $\leqq \boldsymbol{\aleph}_{n}$ has cardinality $\leqq \boldsymbol{\aleph}_{n}$; cl does not increase cardinality by $2.1 ; d_{k} P_{k}(X)$ is $|X|$-generated; and $\left|a\left(X^{\dagger}\right)\right| \leqq|X|$ by (5.3); (i) will be satisfied by $Y_{\alpha}$ if it is satisfied by all $Y_{\beta}$ for $\beta<\alpha$.

Set

$$
Y=\bigcup_{\alpha<N_{n}} Y_{\alpha}
$$

Since each $Y_{\alpha}$ is $u$-closed, so is $Y$. Since $Y_{\alpha+1} \supseteq a\left\{\left[d_{k} P_{k}\left(Y_{\alpha}\right)\right]^{\dagger}\right\}, d_{k} P_{k}\left(Y_{\alpha+1}\right)$ $\supseteq d_{k} P_{k}\left(Y_{\alpha}\right)^{\dagger}$. Hence $d_{k} P_{k}(Y)=\left[d_{k} P_{k}(Y)\right]^{\dagger}$. Moreover, $\left|Y_{\alpha}\right| \leqq \aleph_{n}$ implies $|Y| \leqq$ $\aleph_{n} \aleph_{n}=\aleph_{n}$.

By (iii), $P_{-1}\left(Y_{\beta+1}\right) \supsetneqq P_{-1}\left(Y_{\beta}\right)$. Hence $P_{-1}(Y)=\bigcup_{\alpha<\aleph_{n}} P_{-1}\left(Y_{\alpha}\right)$ is a strictly ascending union of a chain of submodules with order type $\aleph_{n}$. Since any set $A$ of ordinals $<\boldsymbol{\aleph}_{n}$ such that $|A|<\boldsymbol{\aleph}_{n}$ must have a supremum $<\boldsymbol{\aleph}_{n}$, no set of cardinality $<\boldsymbol{\aleph}_{n}$ can generate $P_{-1}(Y)$. Hence $Y$ must satisfy (a), (b), and (c) of the proposition.

6. Quotient fields of regular local rings. In this section, $R$ will denote a commutative domain with quotient field $Q$.

We note that $Q_{R}$ is a directed $R$-module since every cyclic submodule of $Q$ is free and if $a / b, c / d \in Q, 1 / b d \geqq a / b$ and $c / d$. For convenience, we will take as our free generators for $Q$ the set $Q^{\prime}=\{1 / r \mid 0 \neq r \in R\}$ and let $u(1 / r, 1 / s)=1 / r s$. 
6.1 Lemma. Let $R^{\prime}$ be any ring. Then for any projective $R^{\prime}$-module $P, P J\left(R^{\prime}\right) \neq P$.

Proof. See Bass [3, p. 474].

6.2 TheOREM. Let $T$ be a multiplicatively closed subset of $R-\{0\}, T \cap J(R) \neq \varnothing$. Let $M$ be the submodule of $Q$ generated by $T^{-1}=\{1 / t \mid t \in T\}$. Then $\operatorname{hd}_{R}(M)=1 \Leftrightarrow M$ is countably generated.

Proof. Assume $M$ is countably generated. By $4.2, \mathrm{hd}_{R}(M) \leqq 1$. Since $M$ is divisible by some $x \in J(R), \operatorname{hd}_{R}(M) \neq 0$ by 6.1 . Hence $\operatorname{hd}_{R}(M)=1$.

Assume $\operatorname{hd}_{R}(M)=1$. Let $x \in T \cap J(R)$. If $M$ is not countably generated, by 5.2 , we may find a countable multiplicatively closed subset $S$ of $T$ such that $x \in S$ and $d_{1} P_{1}\left(S^{-1}\right)$ is a direct summand of $d_{1} P_{1}\left(T^{-1}\right)$, say $d_{1} P_{1}\left(T^{-1}\right)=d_{1} P_{1}\left(S^{-1}\right) \oplus K$. We apply an argument of Kaplansky [9]. There is a $t \in T$ such that $1 / t \notin P_{-1}\left(S^{-1}\right)$. Then $1 / t \cdot P_{-1}\left(S^{-1}\right) \subseteq M$ and $1 / t \cdot P_{-1}\left(S^{-1}\right) \approx P_{-1}\left(S^{-1}\right)$. Hence

$$
\operatorname{hd}_{R}\left(1 / t \cdot P_{-1}\left(S^{-1}\right)\right)=1 \text {. }
$$

Let

By 3.2, the sequence

$$
Z=1 / t \cdot S^{-1} \cup S^{-1}
$$

$$
P_{0}\left(S^{-1}\right) \oplus P_{1}(Z) \stackrel{I \oplus d_{1}}{\longrightarrow} P_{0}(Z) \stackrel{\nu d_{0}}{\longrightarrow} P_{-1}(Z) / P_{-1}\left(S^{-1}\right) \longrightarrow 0
$$

is exact, so kernel $\nu d_{0}=P_{0}\left(S^{-1}\right)+d_{1} P_{1}(Z)=P_{0}\left(S^{-1}\right) \oplus K \cap d_{1} P_{1}(Z)$ is projective. Hence $\operatorname{hd}_{R}\left(P_{-1}(Z) / P_{-1}\left(S^{-1}\right)\right) \leqq 1$. Since $P_{-1}(Z) / P_{-1}\left(S^{-1}\right)$ is an $R /(t)$ module, by Theorem 1.2 of $[8], P_{-1}(Z) / P_{-1}(Y)$ is $R /(t)$-projective. However $P_{-1}(Z) / P_{-1}\left(S^{-1}\right)$ is a nonzero $R /(t)$ module divisible by $x+(t) \in J(R /(t))$, contradicting 6.1.

6.3 LemmA. Let $R$ be a regular local ring of dimension $n \geqq 2$, and let $\left\{x_{1}, x_{2}, \ldots, x_{n}\right\}$ be a regular system of parameters for $R$. If $\left\{\alpha_{s} \mid s \in R / J\right\}$ is a complete set of coset representatives of $(J,+)$ in $(R,+)$, then $\left\{x_{i+1}+\alpha_{s} x_{i} \mid s \in R / J, 1 \leqq i \leqq n-1\right\}$ are distinct primes in $R$.

Proof. These elements are prime since they are in $J-J^{2}$; they generate distinct ideals since they generate distinct submodules of $J / J^{2}$.

6.4 TheORem. Let $R$ be a regular local ring of dimension $n$. Let $\left\{x_{1}, \ldots, x_{n}\right\}$ be a system of parameters for $R, A \subseteq R / J,|A|=\aleph_{k}$. Let $M$ be generated by a u-closed $M^{\prime} \subseteq Q^{\prime}$ such that $M^{\prime} \supseteq\left\{1 /\left(x_{i+1}-\alpha_{s} x_{i}\right) \mid s \in A, 1 \leqq i \leqq n-1\right\}$ and $\left|M^{\prime}\right|=\aleph_{k}$. Then $\operatorname{hd}_{R}(M)=\min \{n, k+1\}$.

Proof. We note that, if $k=0$ (as it must if $n=1$ ), $\mathrm{hd}_{R}(M) \leqq 1$ by 4.2 , and since $M$ is divisible by some nonunit of $R, \operatorname{hd}_{R}(M) \neq 0$. Hence $\operatorname{hd}_{R}(M)=1=k+1 \leqq n$.

Now assume $k \geqq 1$. Then $\operatorname{hd}_{R}(M) \leqq k+1$ by 4.2 , and $h_{R}(M) \leqq n=$ global dimension of $R$. Hence we need only show both inequalities cannot hold. We use induction on $n$. 
If $n=2, \operatorname{hd}_{R}(M) \neq 1$ by 6.2 , and $M$ is not projective by 6.1 , so $\mathrm{hd}_{R}(M)=2=n$.

Now assume $n \geqq 3$. Let $\mathrm{hd}_{R}(M)=l<n, k+1$. Select a set $A^{\prime} \subseteq A$ with $\left|A^{\prime}\right|=\boldsymbol{\aleph}_{k-1}$ and by 5.2 find a $u$-closed set $Y \subseteq M^{\prime}$ such that $|Y|=\boldsymbol{\aleph}_{k-1}$, no set with fewer elements generates $P_{-1}(Y), Y \supseteq\left\{1 /\left(x_{i+1}-\alpha_{s} x_{i}\right) \mid 1 \leqq i \leqq n-1, s \in A^{\prime}\right\}$, and $d_{l} P_{i}(Y)$ is a direct summand of $d_{l} P_{l}(M)$. Now $\left\{x_{n}-\alpha_{s} x_{n-1} \mid s \in A\right\}$ form a set of primes of cardinality $\boldsymbol{\aleph}_{k}$, so there exists $s^{\prime} \in A$ such that $1 / q=1 /\left(x_{n}-\alpha_{s^{\prime}} x_{n-1}\right)$ is relatively prime to each $1 / y \in Y$. Now $\left\{x_{1}, x_{2}, \ldots, x_{n-1}, q\right\}$ is a regular system of parameters for $R$ so $R^{*}=R /(q)$ is a regular local ring of dimension $n-1$ with $R^{*} / J^{*} \approx R / J$. Also $\left\{x_{i+1}-\alpha_{s} x_{i} \mid 1 \leqq i \leqq n-2, s \in A^{\prime}\right\}$ have exactly the same properties in $R^{*}$ that they had in $R$ (here we are identifying an element in $R$ with its image in $R^{*}$ for convenience).

As above, set $Z=Y \cup\left\{q^{-1} y \mid y \in Y\right\}$. Then $P_{-1}(Z)=q^{-1} P_{-1}(Y)$, so hd ${ }_{R}\left(P_{-1}(Z)\right)$ $=\operatorname{hd}_{R}\left(P_{-1}(Y)\right) \leqq l$. Let $d_{l} P_{l}(Z)=d_{l} P_{l}(Y) \oplus K$. By 3.2 , there is a projective resolution of $P_{-1}(Z) / P_{-1}(Y)$ whose $l$ th image $=\left[\left(-d_{l-1}, I\right) \oplus d_{l}\right]\left[P_{l-1}(Y) \oplus P_{l}(Z)\right]$ $\approx\left(-d_{l-1}, I\right) P_{l-1}(Y) \oplus K \approx P_{l-1}(Y) \oplus K$. Hence $\operatorname{hd}_{R}\left(P_{-1}(Z) / P_{-1}(Y)\right) \leqq l$. As above, $\operatorname{hd}_{R^{*}}\left(P_{-1}(Z) / P_{-1}(Y)\right) \leqq l-1$.

Since $R$ is a unique factorization domain, $Y$ consists of reciprocals of a multiplicative semigroup of $R$, and $q$ is a prime in $R$, for $a / b \in q^{-1} P_{-1}(Y)-P_{-1}(Y)$, $a x / b \in P_{-1}(Y) \Leftrightarrow q \mid x$. Therefore $P_{-1}(Z) / P_{-1}(Y)$ is a torsionless $R^{*}$-module. Since $P_{-1}(Z)$ is generated by $M^{*}=\left\{q^{-1} y^{-1} \mid y^{-1} \in Y\right\}$ and $\left\{y \mid y^{-1} \in Y\right\}$ is a multiplicative semigroup of $R, P_{-1}(Z) / P_{-1}(Y)$ is a directed $R^{*}$-module with upper bound function $u^{*}: M^{*} \times M^{*} \rightarrow M^{*}, u^{*}\left(q^{-1} y^{-1}, q^{-1} z^{-1}\right)=q^{-1} y^{-1} z^{-1}$. Let $u_{1} / u_{2}, v_{1} / v_{2}$ $\in P_{-1}(Z), q \nmid u_{1}, v_{1} ; u_{2}=q u_{3}, v_{2}=q v_{3}$ where $q \nmid v_{3}, u_{3}$. Then

$$
\left(u_{1} / u_{2}\right) \cdot u_{3} v_{1}=\left(v_{1} / v_{2}\right) \cdot v_{3} u_{1} \notin P_{-1}(Y) .
$$

Hence $P_{-1}(Z) / P_{-1}(Y)$ as an $R^{*}$-module is an essential extension of every cyclic submodule, so the map $q^{-1} \rightarrow 1$ extends to an isomorphism between $P_{-1}(Z) / P_{-1}(Y)$ and an $R^{*}$-submodule of $Q^{*}=$ the injective hull of $R^{*}$. Moreover, the image of $M^{*}$ consists of reciprocals of a multiplicative semigroup in $R^{*}$, and if $u \in P_{-1}(Z)$, $y \in Y$, then $u y \in P_{-1}(Z)$. In particular, $u /\left(x_{i+1}-\alpha_{s} x_{i}\right) \in P_{-1}(Z)$ for all $s \in A^{\prime}$, $1 \leqq i \leqq n-2$. Thus $P_{-1}(Z) / P_{-1}(Y)$ as an $R^{*}$-module is divisible by $1 /\left(x_{i+1}-\alpha_{s} x_{i}\right)$ for all $s \in A^{\prime}, 1 \leqq i \leqq n-2$, so $\left\{1 /\left(x_{i+1}-\alpha_{s} x_{i}\right) \mid 1 \leqq i \leqq n-2, s \in A^{\prime}\right\} \subseteq$ the image of $P_{-1}(Z) / P_{-1}(Y)$ in $Q^{*}$.

We now have $R^{*}$ a regular local ring of dimension $n-1,\left|A^{\prime}\right|=\boldsymbol{\aleph}_{k-1}$, and a directed submodule $P_{-1}(Z) / P_{-1}(Y)$ containing $\left\{1 /\left(x_{i+1}-\alpha_{s} x_{i}\right) \mid 1 \leqq i \leqq n-2, s \in A^{\prime}\right\}$. By the induction hypothesis, $\operatorname{hd}_{R^{*}}\left(P_{-1}(Z) / P_{-1}(Y)\right)=\min \{n-1, k\}$. But by [8, Theorem 1.2], $\operatorname{hd}_{R^{*}}\left(P_{-1}(Z) / P_{-1}(Y)\right) \leqq l-1$, where $l<\min \{n, k+1\}$, a contradiction.

6.5 COROLlary. Let $R$ be a regular local ring of dimension $n$ such that a minimal set of generators for $Q$ has cardinality $\leqq|R / J|=\aleph_{k}$. Then $\operatorname{hd}_{R}(Q)=\min \{n, k+1\}$.

Proof. If $n=1, \operatorname{hd}_{R}(Q)=1=\min \{n, k+1\}$. If $n \geqq 2$, we observe that $|Q|=|R|$ $=|R / J|$ by 6.3 . Now apply Theorem 6.4 . 
6.6 COROllaRy. Let $\boldsymbol{R}$ denote the real numbers. Let $\boldsymbol{R}_{n}$ be the localization of $\boldsymbol{R}\left[X_{1}, \ldots, X_{n}\right]$ at the origin, and set $\boldsymbol{Q}_{n}=$ the quotient field of $\boldsymbol{R}_{n}$. Then

$$
\operatorname{hd}_{R_{n}}\left(Q_{n}\right)=n \Leftrightarrow 2 \aleph_{0} \geqq \aleph_{n-1} .
$$

Proof. By $6.5, \operatorname{hd}_{\boldsymbol{R}_{n}}\left(\boldsymbol{Q}_{n}\right)=\min \{n, k+1\}$ where $2 \boldsymbol{\aleph}_{0}=\boldsymbol{\aleph}_{k}$. Hence $\operatorname{hd}_{\boldsymbol{R}_{n}}\left(\boldsymbol{Q}_{n}\right)=n$ $\Leftrightarrow k+1 \geqq n$, i.e. $k \geqq n-1$.

6.7 CoRollary. Let $F$ be a field, $\mathscr{I}$ a nonempty set, $\left\{X_{i} \mid i \in \mathscr{I}\right\}$ algebraically independent elements over $F, R=F\left[\left\{X_{i} \mid i \in \mathscr{I}\right\}\right]$. Let $|\mathscr{I}|=\alpha,|F|=\beta$. Then

$$
\begin{aligned}
\operatorname{hd}_{R}(Q) & =\max (n, k)+1 & \boldsymbol{\aleph}_{0} \beta=\boldsymbol{\aleph}_{k}, & \alpha=\boldsymbol{\aleph}_{n}, \\
& =\min (\alpha, k+1) & \boldsymbol{\aleph}_{0} \beta=\boldsymbol{\aleph}_{k}, & \alpha<\boldsymbol{\aleph}_{0},
\end{aligned}
$$

where an infinite ordinal is replaced by $\infty$.

Proof. Since every element in $R$ is a finite sum of finite products of elements in $\mathscr{I},|R|=\boldsymbol{\aleph}_{0} \alpha \beta$.

Assume $\alpha=\aleph_{n}$. By Small's result (4.2) $h_{R}(Q) \leqq \max (k, n)+1$ since $|Q|=$ $\aleph_{\max (k, n)}$. Let $R_{M}$ be the localization of $R$ at the ideal generated by some set of $m>n+k$ indeterminants. Then $R_{M}$ is a regular local ring of dimension $m$ and $\left|R_{M} / J\left(R_{M}\right)\right|=\aleph_{\max (k, n)}=|R|$. By 6.5, $\operatorname{hd}_{R_{M}}(Q)=\max (k, n)+1$. Since $\operatorname{hd}_{R}(Q)$ $\geqq \mathrm{hd}_{R_{M}}(Q)$, the first case of the theorem follows. (If $\max (k, n)=\infty$, one takes a localization at $m$ indeterminants to get $\mathrm{hd}_{R}(Q) \geqq m$ for all $m$.)

Assume $\alpha<\boldsymbol{\aleph}_{0}$. Then $|R|=\boldsymbol{\aleph}_{0} \beta$. If $\beta<\boldsymbol{\aleph}_{0}$, then $|R|=|Q|=\boldsymbol{\aleph}_{0}$ and by 4.2 , $\operatorname{hd}_{R}(Q)=1=\min (0+1, \alpha)$. So without loss of generality we may assume $\beta \geqq \aleph_{0}$. Then $|R|=|F|$. Let $R^{*}$ be the localization of $R$ at the origin. Then by $6.5, \operatorname{hd}_{R^{*}}(Q)$ $=\min (k+1, \alpha) \leqq \mathrm{hd}_{R}(Q)$. But the global dimension of $R=\alpha$, so $\mathrm{hd}_{R}(Q) \leqq \alpha$ and $|Q|=\aleph_{k}$ so $\operatorname{hd}_{R}(Q) \leqq k+1$. Hence $\mathrm{hd}_{R}(Q)=\min (k+1, n)$.

6.5 and 6.7 can be generalized slightly. Let $R=K\left[x_{1}, \ldots, x_{m}\right]$ be a finitely generated ring extension of an infinite field $K$ (the $x_{i}$ not necessarily indeterminants). Assume $R$ has global dimension $n<\infty$. By Auslander and Buchsbaum [2], some localization of $R$ at a maximal ideal, say $R_{M}$, has codimension $n$. Since the global dimension of $R_{M} \leqq$ the global dimension of $R, R_{M}$ is a regular local ring of dimension $n$. (See Kaplansky [8].)

6.8 Corollary. Let $R=K\left[x_{1}, \ldots, x_{m}\right]$ have global dimension $n<\infty$, and let $|K|=\aleph_{k}$. Then $\operatorname{hd}_{R}(Q)=\min \{n, k+1\}$.

Proof. Clearly $\mathrm{hd}_{R}(Q) \leqq n$ and by $4.2 \mathrm{hd}_{R}(Q) \leqq k+1$. Let $R_{M}$ be a localization of $R$ of dimension $n$. Then $\operatorname{hd}_{R}(Q) \geqq \operatorname{hd}_{R_{M}}(Q)=\min \{k+1, n\}$ since $\left|R_{M}\right|=|R|$ $=|K|=\aleph_{k}=|R / J|$. Hence $\operatorname{hd}_{R}(Q)=\min \{k+1, n\}$.

6.9 Corollary. Let $R=K\left[x_{1}, \ldots, x_{m}\right]$. Assume for all positive integers $l$ there exists a prime ideal $M \subseteq R$ such that $l \leqq \mathrm{gl}$. $\mathrm{d}\left(R_{M}\right)<\infty$. If $|K|=\aleph_{h}$, then $\operatorname{hd}_{R}(Q)$ $=h+1$ if $h \in \omega, \infty$ otherwise. 
Proof. If $h \in \omega$, there is an $M$ with $h+2 \leqq \mathrm{gl}$. $\mathrm{d}\left(R_{M}\right)<\infty$. Then $R_{M}$ is regular and $\operatorname{hd}_{R_{M}}(Q)=h+1 \leqq \mathrm{hd}_{R}(Q)$. By 4.2, $\mathrm{hd}_{R}(Q)=h+1$.

If $h \notin \omega$, for all $l \in \omega$ there is an $M$ with $l \leqq \operatorname{hd}_{R_{M}}(Q) \leqq \operatorname{hd}_{R}(Q)$. Hence hd $\operatorname{lo}_{R}(Q)=\infty$.

In proving 6.4 , we needed some property which would enable us to use induction from dimension $n$ to dimension $n-1$ without having a collapse in the number of generators of $P_{-1}(Z) / P_{-1}(Y)$. We chose a situation which yielded the desired result when a set of cardinality $|R / J|$ generated $Q$. On the opposite end of the scale are complete regular local rings. Here too we can calculate $\mathrm{hd}_{R}(Q)$. In the case that the characteristics of $R$ and $R / J$ are equal, these are just power series over a field (see [15, p. 307]). In the nonequicharacteristic case they are still close enough to power series to keep track of cardinality of a special set of primes.

6.10 TheOREM. Let $R$ be a complete regular local ring of dimension $n$. Let $Q$ be generated by $\aleph_{k}$ but no fewer elements. Then $\operatorname{hd}_{R}(Q)=\min (n, k+1)$.

Proof. Let $|R / J|=\alpha$. Since $J^{i} / J^{i+1}$ is a finitely generated $R / J$-module, $\left|J^{i} / J^{i+1}\right|$ $=|R / J|$. Hence $\left|R / J^{i+1}\right|=|R / J|$. Now the elements of $R$ are limits of Cauchy sequences in $R$, so each is completely determined by its sequence of projections in $R / J^{i+1}$. Moreover there are $|R / J|$ different ways two Cauchy sequences agreeing in $R / J^{i}$ can differ in $R / J^{i+1}$. Hence $|R|=|R / J|^{\aleph_{0}}$. Now assume $n \geqq 2$. Let $\left\{x_{1}, x_{2}\right.$, $\left.\ldots, x_{n}\right\}$ be a system of parameters for $R$. There are $|R / J| \aleph_{0}$ Cauchy sequences $\left\{y_{i}\right\}$ in $x_{1}$. For each of these, let $\alpha_{\left\{y_{i}\right\}}$ be the element it determines. Any element of the form $x_{2}-x_{1} \alpha_{\left\{y_{1}\right\}}$ is prime since it belongs to $J$ but not $J^{2}$ (its projection on $J / J^{2}=x_{2}-x_{1} k$ for some $\left.k \in R\right)$. Let $x_{2}-x_{1} \alpha_{\left\{y_{i}\right\}}=\left(x_{2}-x_{1} \alpha_{\left\{z_{i}\right\}}\right) \gamma$ where $\gamma$ is a unit. Then $x_{2}(1-\gamma)=x_{1}\left(\alpha_{\left\{y_{i}\right\}}-\alpha_{\left\{z_{i}\right\}} \gamma\right)$. Since $x_{1} \nmid x_{2}, x_{1} \mid 1-\gamma$, so $\gamma=1+x_{1} r$. Assume $\gamma=1+x_{1}^{n} r_{n}$. Then $\alpha_{\left\{y_{i}\right\}}-\alpha_{\left\{z_{i}\right\}}\left(1+x_{1}^{n} r_{n}\right)=\alpha_{\left\{y_{i}-z_{i}\right\}}-\alpha_{\left\{z_{i}\right)} x_{1}^{n} r_{n}$. Since $x_{2}(1-\gamma) \in J^{n+1}$, $\left(\alpha_{\left\{y_{i}-z_{i}\right\}}-\alpha_{\left\{z_{i}\right\}} x_{1}^{n} r_{n}\right) \in J^{n}$. Hence $\alpha_{\left\{y_{i}-z_{i}\right\}} \in J^{n} . \alpha_{\left\{y_{i}-z_{i}\right\}}$ is a Cauchy sequence in $x_{1}$ which lies in $J^{n}$, hence $x_{1}^{n} \mid \alpha_{\left\{y_{i}-z_{i}\right\}}$, and so $x_{1}^{n} \mid\left(\alpha_{\left\{y_{i}-z_{i}\right\}}-\alpha_{\left\{z_{i}\right\}} x_{1}^{n} r_{i}\right)$ so $x_{1}^{n+1} \mid 1-\gamma$ for all $n$. This contradicts the unique factorization property of $R$. We thus have $|R|$ distinct primes in $R$ of the form $x_{i+1}-f\left(x_{i}\right)$ which remain distinct in $R /\left(x_{n}\right)$ for $n \geqq 3$. The proof of the theorem now proceeds exactly as in 6.4 with these $|R|$ primes replacing $\left\{1 /\left(x_{i+1}-\alpha_{s} x_{i}\right)\right\}$.

Note that, if $|R / J|=|R / J| \aleph_{0}$, since any local ring is a subring of its completion, $|R|=|R / J|$ so 6.5 applies to the ring $R$. Moreover, if $R$ is any regular local ring with $|R / J| \geqq \aleph_{\omega}$, then $\operatorname{hd}_{R}(Q)=$ the dimension of $R$ since 5.2 enables us to find a directed module $M \subseteq Q$ containing $\boldsymbol{\aleph}_{\mathrm{dm} R+1}$ elements of the form $1 /\left(x_{i+1}-\alpha x_{i}\right)$ such that $\operatorname{hd}_{R}(M) \leqq \operatorname{hd}_{R}(Q)$. We then apply 6.4 .

7. Directed modules with linearly ordered free generating sets. In this section we generalize results in Osofsky [13].

7.1. Lemma. Let $M$ be a directed module with a free generating set $M^{\prime}$ and upper bound function $u$. Assume no set of cardinality $\leqq \aleph_{n}$ generates $M$ and every set 
$X \subseteq M^{\prime}$ with $|X|=\aleph_{n}$ has an upper bound in $M^{\prime}$. If $\operatorname{hd}_{R}(M) \leqq k$, then there is a $u$-closed subset $Y \subseteq M^{\prime}$ such that the smallest cardinality of a generating set for $P_{-1}(Y)$ is $\aleph_{n}$ and $\operatorname{hd}_{R}\left(P_{-1}(Y)\right) \leqq k-1$.

Proof. By 5.2, there exists a $u$-closed set $Y$ of cardinality $\boldsymbol{\aleph}_{n}$ such that no set of cardinality $<\aleph_{n}$ generates $P_{-1}(Y)$ and $d_{k} P_{k}(Y)$ is a direct summand of $d_{k} P_{k}\left(M^{\prime}\right)$. Let $z$ be an upper bound for $Y$. Then

$$
P_{k-1}\left(M^{\prime}\right)=P_{k-1}(Y) \oplus \sum_{\text {some } x_{i} \notin Y}\left\langle x_{0}, \ldots, x_{k-1}\right\rangle R .
$$

We may subtract any element in the second sum from each free generator of $P_{k-1}(Y)$ and still have a direct sum. In particular,

$$
P_{k-1}\left(M^{\prime}\right)=d_{k}\left[z^{*} P_{k-1}(Y)\right] \oplus \sum_{\text {some } x_{i} \notin Y}\left\langle x_{0} \cdots x_{k-1}\right\rangle R
$$

and

$$
d_{k} P_{k}(Y) \subseteq d_{k}\left[z^{*} P_{k-1}(Y)\right] \subseteq d_{k} P_{k}\left(M^{\prime}\right)
$$

Hence $d_{k} P_{k}(Y)$ is a direct summand of a direct summand of $P_{k-1}\left(M^{\prime}\right)$. We then have $d_{k} P_{k}(Y)$ a direct summand of $P_{k-1}(Y)$, so $d_{k-1} P_{k-1}(Y)$ is projective.

7.2 Lemma (Dual basis lemma). Let $R$ be any ring, $P$ a right $R$-module. Then $P$ is projective $\Leftrightarrow$ there exists $\left\{x_{i} \mid i \in \mathscr{I}\right\} \subseteq P$ and $\left\{f_{i} \mid i \in \mathscr{I}\right\} \subseteq \operatorname{Hom}_{R}(P, R)$ such that for all $x \in P, f_{i}(x)=0$ for all but a finite number of $i \in \mathscr{I}$, and $x=\sum_{i \in \mathscr{I}} x_{i} f_{i}(x)$.

For a proof see Cartan and Eilenberg [2, p. 132].

7.3 Lemma. Let $R$ be a ring with no zero divisors, $M=\bigcup_{i=1}^{\infty} x_{i} R$ an $R$-module, $x_{i} R \supsetneqq x_{j} R$ for all $i \supsetneqq j$. Then $M$ is not projective.

Proof. Let $f: M \rightarrow R, f \neq 0$. Then there exists $i$ such that $f\left(x_{i}\right) \neq 0$. Let $0 \neq x \in M$. Then there exists $j \geqq i$ such that $x=x_{j} r \in x_{j} R$. Since $x_{i} \in x_{j} R, f\left(x_{j}\right) \neq 0$ and $f(x)=$ $f\left(x_{j}\right) r \neq 0$ since $R$ has no zero divisors. Since $M$ is a union of proper submodules, $M$ cannot be finitely generated. Hence the dual basis property in 7.2 cannot hold for $M$.

7.4 TheORem. Let $R$ be a ring with no zero divisors, $M$ a directed $R$-module with a linearly ordered set of free generators $M^{\prime}$. Then $\mathrm{hd}_{R}(M)=n+1 \Leftrightarrow$ the smallest cardinality of a generating set for $M$ is $\aleph_{n}$.

Proof. If $M$ is cyclic, $\operatorname{hd}_{R}(M)=0$, and if $M$ is countably generated but not cyclic, $\operatorname{hd}_{R}(M) \geqq 1$ by 7.3. Now assume the theorem for $n-1$. By $4.2, \operatorname{hd}_{R}(M) \leqq n+1$. The linear ordering on $M^{\prime}$ insures upper bounds for all sets of cardinality $<\aleph_{n}$. By 7.1, there exists a $u$-closed subset $Y \subseteq M^{\prime}$ such that the smallest cardinality of a generating set for $P_{-1}(Y)$ is $\aleph_{n-1}$ and $\operatorname{hd}_{R}\left(P_{-1}(Y)\right) \leqq$ hd $R(M)-1$. By the induction 
hypothesis, $\operatorname{hd}_{R}\left(P_{-1}(Y)\right)=n$, so $\mathrm{hd}_{R}(M) \geqq n+1$. If $\Rightarrow$ fails, 7.1 yields an $\boldsymbol{\aleph}_{n}$ generated $P_{-1}(Y)$ of dimension $\leqq n$, contradicting the induction hypothesis.

7.5 COROLlary. Let $R$ be a ring with no zero divisors and linearly ordered right ideals. If every ideal of $R$ is finitely generated, the global dimension of $R=0$ or 1 . Otherwise, the global dimension of $R=2+\sup \{n \mid R$ possesses an ideal generated by $\aleph_{n}$ but no fewer elements\}.

Proof. This is an immediate consequence of 7.4 and the global dimension theorem (Auslander [1]).

7.6 Corollary. Let $R$ be a ring with no zero divisors possessing a linearly ordered set of left ideals $\left\{R x_{i} \mid i \in \mathscr{I}\right\}$ such that for all $y \in R$ there is an $i \in \mathscr{I}$ with $R x_{i} \subseteq R y$. Let $Q$ be the left quotient ring of $R$. Then $\mathrm{hd}_{R}(Q)=n+1$, where $\aleph_{n}$ is the cardinality of a smallest generating set for $Q_{R}$.

Proof. By hypothesis, $R$ must be a left Ore domain so $Q=\left\{x^{-1} r \mid 0 \neq x \in\right.$ $R, r \in R\}$. For $y \in R$, let $r y=x_{i}$. Then $y^{-1}=x_{i}^{-1} r$, so $\left\{x_{i}^{-1} \mid i \in \mathscr{I}\right\}$ generate $Q_{R}$, and they are linearly ordered by $\leqq$. We now apply 7.4 .

8. Appendix. Cohen has shown [6] that it is consistent to assume that $2 \mathrm{~N}_{0}=\kappa$ for any cardinal $\kappa$ which is not a countable union of smaller cardinals. By Corollary 6.7, if $\boldsymbol{R}=$ the real numbers and $R=\boldsymbol{R}\left[X_{1}, \ldots, X_{n}\right]$ for $n \geqq 3$, then $\mathrm{hd}_{R}(Q)=k<n$ $\Leftrightarrow 2 \aleph_{0}=\aleph_{k-1}$. In particular, $\operatorname{hd}_{R}(Q)=2 \Leftrightarrow$ the continuum hypothesis holds. This is true $\Leftrightarrow d_{2} P_{2}\left((R-\{0\})^{-1}\right)$ is projective and by a result of Bass [4], since $d_{2} P_{2}\left((R-\{0\})^{-1}\right)$ is infinitely generated, it is projective $\Leftrightarrow$ it is free. In this appendix, we construct a free basis for $d_{2} P_{2}\left(M^{\prime}\right)$ for any directed module with $\left|M^{\prime}\right|=\boldsymbol{\aleph}_{1}$. Thus one can show constructively that $2^{\aleph_{0}}=\aleph_{1} \Rightarrow d_{2} P_{2}\left((R-\{0\})^{-1}\right)$ has a free basis. The reverse implication, however, depends on the number of variables in the polynomial ring. If $S=R[X, Y], d_{2} P_{2}\left((S-\{0\})^{-1}\right)$ always has a free basis, regardless of the cardinality of $\boldsymbol{R}$.

For the rest of this appendix, $M$ will denote a directed module with free generators $M^{\prime}$.

A.1 Lemma. Assume $M$ is countably generated. Then $d_{1} P_{1}\left(M^{\prime}\right)$ has a free basis of the form $\left\{d_{1}\langle a, b\rangle\right\}$.

Proof. Since $M$ is countably generated, there exist $\left\{x_{i} \mid i \in \omega\right\} \subseteq M^{\prime}$ such that $x_{0}<x_{1}<\cdots$ and $M=\sum_{i=0}^{\infty} x_{i} R$. For each $y \in M^{\prime}$, let $x(y)$ denote the $x_{i}$ with smallest index $i$ such that $y \in x_{i} R$. We show $\left\{d_{1}\langle x(y), y\rangle \mid y \in M^{\prime}\right\}$ is a free basis for $d_{1} P_{1}\left(M^{\prime}\right)$.

Let $\sum_{j=1}^{n} d_{1}\left\langle x\left(y_{j}\right), y_{j}\right\rangle r_{j}=0$, all $r_{j} \neq 0$, and assume $x\left(y_{n}\right)=x_{k}$ is the largest $x_{i}$ occurring. Since $r_{n} \neq 0, x\left(y_{n}\right)$ must appear in another pair $\left\langle x_{k}, y_{j}\right\rangle$, so in at least one of its appearances, the second component $y_{j} \neq x_{k-1}$. But then $\left\langle y_{j}\right\rangle r_{j}$ is a term of $d_{1}\left\langle x_{k}, y_{j}\right\rangle$ but of no other $d_{1}\left\langle x\left(y_{l}\right), y_{l}\right\rangle$, so the sum cannot be zero. 
Let $\langle a, b\rangle$ be a generator for $P_{1}\left(M^{\prime}\right)$. Then $d_{2}\langle x(a), a, b\rangle=\langle a, b\rangle-\langle x(a), b\rangle$ $+\langle x(a), a\rangle a^{-1} b$ and since $\mathscr{P}_{M^{\prime}}$ is a complex,

$$
d_{1}\langle a, b\rangle=d_{1}\langle x(a), b\rangle-d_{1}\langle x(a), a\rangle a^{-1} b .
$$

Let $x(a)=x_{l}, x(b)=x_{k}$. Then

$$
d_{1}\langle x(a), b\rangle=d_{1}\langle x(b), b\rangle+\sum_{i=k}^{l-1} d_{1}\left\langle x_{i+1}, x_{i}\right\rangle x_{i}^{-1} b .
$$

Thus every generator for $d_{1} P_{1}\left(M^{\prime}\right)$ may be expressed as a linear combination of elements in the given set.

A.2 Proposition. If $M$ is $\aleph_{1}$-generated, then $d_{2} P_{2}\left(M^{\prime}\right)$ is free.

Proof. Since $M$ is $\boldsymbol{\aleph}_{1}$-generated, there exist $u$-closed subsets $\left\{T_{\alpha} \mid \alpha<\boldsymbol{\aleph}_{1}\right\}$ $\subseteq M^{\prime}$ such that $M^{\prime} \cap P_{-1}\left(T_{\alpha}\right) \subseteq T_{\alpha}$ for all $\alpha, P_{-1}\left(T_{\alpha}\right)$ is countably generated, $T_{\alpha} \subset T_{\beta}$ for $\alpha<\beta$, and $M=\bigcup_{\alpha<\aleph_{1}} P_{-1}\left(T_{\alpha}\right)$. Set $T_{-1}=\varnothing$. It is sufficient to show

$$
d_{2} P_{2}\left(T_{\alpha}\right) / d_{2} P_{2}\left(T_{\alpha-1}\right)
$$

has a free basis for all $\alpha<\boldsymbol{\aleph}_{1}, \alpha$ a successor ordinal.

By the conditions on the $T_{\alpha}$, there exist $\left\{x_{\mathfrak{i}} \mid i \in \omega\right\} \subseteq T_{\alpha}-T_{\alpha-1}$ such that $P_{-1}\left(T_{\alpha}\right)$ $=\bigcup_{i=0}^{\infty} x_{i} R$. For $z \in T_{\alpha-1}, y \in T_{\alpha}$, define $x_{\alpha-1}(z)$ and $x_{\alpha}(y)$ as in A.1. Then

$$
\begin{aligned}
P_{1}\left(T_{\alpha}\right)= & \sum_{y \in T_{\alpha}}\left\langle x_{\alpha}(y), y\right\rangle R \oplus \sum_{z \in T_{\alpha-1}}\left\langle x_{\alpha-1}(z), z\right\rangle R \oplus \sum_{u \in T_{\alpha-1} ; u \neq x_{\alpha-1}(v)}\langle u, v\rangle R \\
& \oplus \sum_{a \in T_{\alpha}-T_{\alpha-1} ; a \neq x_{\alpha}(b)}\langle a, b\rangle R .
\end{aligned}
$$

For each $\langle a, b\rangle$ with $a=x_{\alpha-1}(b)$ or $a \in T_{\alpha}-T_{\alpha-1}$ and $a \neq x_{\alpha}(b)$ there exists a unique element $p=\sum\left\langle x_{\alpha}\left(y_{i}\right), y_{i}\right\rangle r_{i}$ such that $d_{1}\langle a, b\rangle=d_{1} p$. Then $\langle a, b\rangle-p=d_{2} q_{a, b}$ for some $q_{a, b} \in P_{2}\left(T_{\alpha}\right)-P_{2}\left(T_{\alpha-1}\right)$. Then $\mathfrak{F}=\left\{d_{2} q_{a, b} \mid a=x_{\alpha-1}(b)\right.$ or $a \in T_{\alpha}-T_{\alpha-1}$, $\left.a \neq x_{\alpha}(b)\right\}$ is a free basis for $d_{2} P_{2}\left(T_{\alpha}\right) / d_{2} P_{2}\left(T_{\alpha-1}\right)$.

$\mathfrak{F}$ is independent since $\{\langle a, b\rangle\}$ is independent in $P_{2}\left(T_{\alpha}\right)$ modulo the first and third sums, and the image of $d_{2} q_{a, b}=$ the image of $\langle a, b\rangle$ in that module.

To show $\mathfrak{F}$ spans, we need only show that, for all $u \in T_{\alpha}-T_{\alpha-1}, d_{2}\langle u, v, w\rangle$ is a linear combination of elements in $\mathfrak{F}$ and an element in $d_{2} P_{2}\left(T_{\alpha-1}\right)$.

$$
d_{2}\langle u, v, w\rangle=\langle v, w\rangle-\langle u, w\rangle+\langle u, v\rangle v^{-1} w
$$

Let

$$
\begin{aligned}
& \bar{q}_{a, b}=q_{a, b} \quad a=x_{\alpha-1}(b) \quad \text { or } \quad a \in T_{\alpha}-T_{\alpha-1}, a \neq x_{\alpha}(b), \\
& =0 \quad \text { otherwise. }
\end{aligned}
$$

If $q_{v, w}$ is defined or $v=x_{\alpha}(w)$, consider $d_{2}\left(\langle u, v, w\rangle-\bar{q}_{v, w}+\bar{q}_{u, w}-\bar{q}_{u, v} v^{-1} w\right)$. This is an element of $\sum_{y \in T_{\alpha}}\left\langle x_{\alpha}(y), y\right\rangle R$ in the kernel of $d_{1}$. By A.1, it must be 0 , so

$$
d_{2}(\langle u, v, w\rangle)=d_{2} \bar{q}_{v, w}-d_{2} \bar{q}_{u, w}+d_{2} \bar{q}_{u, v} v^{-1} w .
$$


If $v \in T_{\alpha-1}, v \neq x_{\alpha-1}(w)$, we apply A.1 to $d_{1} P_{1}\left(T_{\alpha-1}\right)$ to express $d_{1}\langle v, w\rangle$ uniquely as a sum $\sum d_{1}\left\langle x_{\alpha-1}\left(y_{i}\right), y_{i}\right\rangle r_{i}$. Then $p=\langle v, w\rangle-\sum\left\langle x_{\alpha-1}\left(y_{i}\right), y_{i}\right\rangle \boldsymbol{r}_{i} \in d_{2} P_{2}\left(T_{\alpha-1}\right)$ and $d_{2}\langle u, v, w\rangle=-d_{2} \bar{q}_{u, w}+d_{2} \bar{q}_{u, v} v^{-1} w+p+\sum d_{2} q_{x_{\alpha-1}\left(y_{i}\right), y_{i}} r_{i}$.

We note that if $M$ is countably generated, this construction yields a basis for $d_{2} P_{2}\left(M^{\prime}\right)$ by taking a smaller union (or even setting $M^{\prime}=T_{0}$ ).

In the above construction of a free basis, the ring never appears-just the generators $M^{\prime}$. This is not surprising in view of 4.2-the ring is not used in that proof either. By 6.7 or 7.4 it is impossible to find a free basis for $d_{2} P_{2}\left(M^{\prime}\right)$ expressed only in terms of elements of $M^{\prime}$ if $M$ is not at most $\aleph_{1}$-generated. If there is no 1-1 map between $R=R[X, Y]$ and $\boldsymbol{N}_{1}$, the free basis for $d_{2} P_{2}(Q-\{0\})$, which exists since gl. d. $R=2$ and infinite $R$-projectives are free, must depend heavily on the ring $R$. If one adds another variable to get $R^{\prime}=R[X, Y, Z]$, the description of $d_{2} P_{2}\left(Q^{\prime}-\{0\}\right)$ is formally the same but no free basis exists.

\section{REFERENCES}

1. M. Auslander, On the dimension of modules and algebras. III, Nagoya Math. J. 9 (1955), 67-77.

2. M. Auslander and D. Buchsbaum, Homological dimension in Noetherian rings. II, Trans. Amer. Math. Soc. 88 (1958), 194-206.

3. H. Bass, Finite dimension and a homological generalization of semi-primary rings, Trans. Amer. Math. Soc. 95 (1960), 466-488.

4. _- Big projective modules are free, Illinois J. Math. 7 (1963), 23-31.

5. H. Cartan and S. Eilenberg, Homological algebra, Princeton Univ. Press, Princeton, N. J., 1956.

6. P. J. Cohen, Set theory and the continuum hypothesis, Benjamin, New York, 1966.

7. I. Kaplansky, Projective modules, Ann. of Math. (2) 68 (1958), 372-377.

8. - Homological dimension of rings and modules, Mimeographed notes, Univ. of Chicago, Illinois, 1959.

9. - The homological dimension of a quotient field, Nagoya Math. J. 27 (1966), 139-142.

10. E. Matlis, Divisible modules, Proc. Amer. Math. Soc. 11 (1960), 385-391.

11. - Cotorsion modules, Mem. Amer. Math. Soc. No. 49 (1964).

12. M. Nagata, Local rings, Interscience Tracts in Pure and Applied Math., No. 13, Interscience, New York, 1962.

13. B. Osofsky, Global dimension of valuation rings, Trans. Amer. Math. Soc. 126 (1967), 136-149.

14. L. Small, Some remarks on the homological dimension of a quotient field, Mimeographed notes, Univ. of California, Berkeley, Calif., 1966.

15. O. Zariski and P. Samuel, Commutative algebra, Vol. II, Van Nostrand, Princeton, N. J., 1960.

Rutgers-The State University,

New BRUnSWick, NeW JeRSEY 